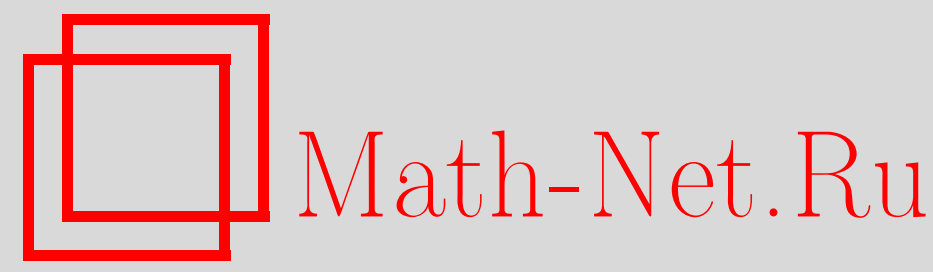

В. А. Клячин, В. М. Миклюков, Признаки неустойчивости поверхностей нулевой средней кривизны в искривленных лоренцевых произведениях, Матем. сб., 1996, том 187, номер 11, 67-88

DOI: https://doi.org/10.4213/sm172

Использование Общероссийского математического портала Math-Net.Ru подразумевает, что вы прочитали и согласны с пользовательским соглашением

http://www.mathnet.ru/rus/agreement

Параметры загрузки:

IP : 3.85 .7 .115

26 апреля 2023 г., 15:11:21 


\title{
Признаки неустойчивости поверхностей нулевой средней кривизны в искривленных лоренцевых произведениях
}

\begin{abstract}
В работе исследуется устойчивость поверхностей нулевой средней кривизны в лоренцевых многообразиях. В случае, когда объемлющее многообразие является искривленным лоренцевым произведением и при некоторых предположениях относительно искривляющей функции, доказьвается, что всякая устойчивая минимальная трубка или лента является вполне геодезическим многообразием.

Библиографияя: 22 названия.
\end{abstract}

\section{Введение}

В настоящей работе исследуется проблема устойчивости подмногообразий нулевой средней кривизны и произвольной коразмерности в псевдоримановых многообразиях (см., например, [1]).

Проблема устойчивости подмногообразий нулевой средней кривизны в римановых многообразиях изучена весьма глубоко. Только в последние годы этой проблеме были посвящены статьи Барбосы и до Кармо, Лоусона, А.В. Погорелова, Саймонса, А. А. Тужилина, А. Т. Фоменко и др.

Соответствующая проблема в псевдоримановых многообразиях практически не исследована. Предпринятый нами информационный поиск позволил обнаружить лишь две пионерские работы, непосредственно связанные с данным кругом вопросов. Так, в статье В.П. Гороха [2] доказана локальная максимальность пространственноподобных графиков нулевой средней кривизны, а в случае времениподобных поверхностей нулевой средней кривизны в $R_{1}^{3}$ установлено сушествование локальных вариаций как уменьшающих, так и увеличивающих площадь. В статье Шена [3] показано, что всякое пространственноподобное подмногообразие $M$ с нулевым вектором средней кривизны в псевдоримановом многообразии неотрицательной секционной кривизны является устойчивым, и что если объемлющее многообразие является полным односвязным с постоянной неотрицательной кривизной и $M$ полное, то $M$ есть вполне геодезическое подмногообразие.

Подмногообразия нулевой средней кривизны суть экстремали функционала объема (первая вариация функционала объема обращается в нуль).

В римановом случае устойчивость означает положительную определенность второй вариации функционала объема, и каждое подмногообразие с нулевьм вектором средней кривизны устойчиво в достаточно малой окрестности любой своей точки.

Работа вьполнена при финансовой поддержке Российского фонда фундаментальных исследований (грант № 93-011-176). 
В псевдоримановом случае локальная устойчивость представляет собой существенно более тонкое свойство. Так, например, в случае пространственноподобных поверхностей нулевой средней кривизны в лоренцевых многообразиях малые (по носителю и амплитуде) вариации в направлении времениподобного сечения нормального расслоения не увеличивают функционала объема, а в направлении пространственноподобного сечения - не уменьшают функционала объема. Соответственно, вопросы глобальной устойчивости также являются значительно более сложньгми.

Ниже рассматривается специальный частный случай псевдоримановых многообразий - лоренцевы многообразия. При этом главное внимание уделяется ситуации, в которой объемлющее многообразие является искривленным лоренцевым произведением, т.е. диффеоморфно декартову произведению $H \times \mathbb{R}$ некоторого риманова многообразия $H$ с метрикой $d s_{H}^{2}$ на вешественную прямую $\mathbb{R}$ с евклидовой метрикой $d t^{2}$, причем псевдометрика $d s^{2}$ на $H$ определяется следующим образом: $d s^{2}=-\delta(x) d t^{2}+d s_{H}^{2}$, где $\delta(x)>0$ - некоторая, наперед заданная на $H$ функция, $x \in H$.

Основными объектами наших исследований являются трубки и ленты нулевой средней кривизны в искривленных лоренцевых произведениях. Данные классы поверхностей тесно связаны с релятивистской струной и имеют вполне конкретный физический смысл (см., например, [4] и цитированную там литературу).

Известно [5], что всякая устойчивая минимальная гиперповерхность параболического типа в римановом многообразии неотрицательной кривизны Риччи является вполне геодезическим подмногообразием. В настояшей работе данный результат распространяется на случай пространственноподобных поверхностей в искривленном лоренцевом произведении $H \times_{\delta} \widehat{\mathbb{R}}$.

При некоторых предположениях относительно искривляющей функции $\delta(x)$ доказывается, что всякая устойчивая минимальная трубка или лента "в целом", имеющая нулевую среднюю кривизну в $H \times_{\delta} \widehat{\mathbb{R}}$, является вполне геодезическим подмногообразием.

Строятся примеры поверхностей нулевой средней кривизны, иллюстрирующие полученные результаты.

Указываются условия, при выполнении которых ленты нулевой средней кривизны являются плоскими (или частью геликоида).

\section{1. Общее уравнение для второй вариации}

Пусть $N-(n+1)$-мерное связное многообразие класса $C^{2}$. Многообразие $N$ называется лорениевым, если на нем задано невырожденное гладкое симметричное тензорное поле типа $(0,2)$ сигнатуры $(1, n)$ (см. [6, с. 23]), называемое скалярным произведением и обозначаемое через $\langle$,$\rangle .$

Пусть $z \in N, X \in T_{z} N$, где $T_{z} N$ - касательное пространство к $N$ в точке $z$. Скалярный квадрат вектора $X$ обозначается через $|X|^{2}$. Ненулевой вектор $X \in T_{z} N$ называется пространственноподобным, светоподобным или времениподобным в зависимости от того, что $|X|^{2}>0,|X|^{2}=0$ или $|X|^{2}<0$.

Для заданного метрического тензора на $N$ существует единственная согласованная с ним связность Леви-Чивита [6, с. 360], которую мы будем обозначать через $\bar{\nabla}$.

Пусть $M-p$-мерное, некомпактное, связное, ориентируемое $C^{2}$-многообразие с 
кусочно-гладким краем $\partial M$ (случай $\partial M=\varnothing$ не исключается). Рассмотрим поверхность $\mathscr{M}=(M, u)$, заданную $C^{2}$-погружением $u: M \rightarrow N, 2 \leqslant p \leqslant n$. Поверхность $\mathscr{M}$ называется пространственноподобной, если каждый касательный к ней вектор пространственноподобен. Будем называть поверхность $\mathscr{M}$ времениподобной, если в каждой точке существует хотя бы один времениподобный касательный к ней вектор.

Связность $\bar{\nabla}$ индуцирует соответствующую связность $\nabla$ на поверхности $\mathscr{M}$. Для произвольных $C^{1}$-гладких векторных полей $X, Y$ на $\mathscr{M}$ и функции $h \in C^{1}(M)$ связность $\nabla$ определяется следующим образом. Рассматриваются произвольные $C^{1}$ продолжения векторных полей $X, Y$ и функции $h$ в некоторую окрестность поверхности $\mathscr{M}$. Тогда

$$
\nabla h=(\bar{\nabla} h)^{T}, \quad \nabla_{X} Y=\left(\bar{\nabla}_{X} Y\right)^{T}
$$

где $(v)^{T}$ - ортогональная проекция вектора $v$ на касательное пространство $T_{u(m)} \mathscr{M}$.

Пусть $m \in M$ и пусть в некоторой окрестности точки $u(m)$ определены гладкие векторные поля $X$ и $Y$. Билинейная форма

$$
B(X(m), Y(m))=\left(\bar{\nabla}_{X} Y\right)(u(m))-\left(\bar{\nabla}_{X} Y\right)^{T}(u(m))
$$

называется второй фундаментальной формой поверхности $\mathscr{M}[7$, с. 56]. Если $\left\{E_{i}\right\}_{i=1}^{p}-$ ортонормированный базис в касательном пространстве к поверхности $\mathscr{M}$ в точке $u(m)$, то вектор

$$
H(m)=\frac{1}{p} \operatorname{trace} B=\frac{1}{p} \sum_{i=1}^{p} B\left(E_{i}, E_{i}\right) \delta_{i}, \quad \delta_{i}=\left|E_{i}\right|^{2},
$$

называется вектором средней кривизны поверхности $\mathscr{M}$ в точке $u(m)$.

Пусть $N_{u(m)} \mathscr{M}$ - нормальное пространство к поверхности $\mathscr{M}$ в точке $u(m)$. Для произвольного вектора $v \in N_{u(m)} \mathscr{M}$ пусть $A^{v}$ означает гомоморфизм Вейнгартена, определяемый как линейное преобразование $A^{v}: T_{u(m)} \mathscr{M} \rightarrow T_{u(m)} \mathscr{M}$, двойственное к билинейной форме $B$

$$
\left\langle A^{v}(X), Y\right\rangle=\langle B(X, Y), v\rangle
$$

Положим

$$
\begin{gathered}
\left\|A^{v}\right\|^{2}=\sum_{i=1}^{p} \delta_{i}\left|A^{v}\left(E_{i}\right)\right|^{2} \\
k(v)=\sum_{i=1}^{p} \delta_{i}\left\langle\bar{R}\left(v, E_{i}\right) v, E_{i}\right\rangle, \quad \delta_{i}=\left|E_{i}\right|^{2}
\end{gathered}
$$

где $\bar{R}$ - тензор кривизны многообразия $N$ и $\left\{E_{i}\right\}_{i=1}^{p}-$ ортонормированньй базис в $T_{u(m)} \mathscr{M}$.

Пусть $v$ - сечение нормального расслоения поверхности $\mathscr{M}$. Продолжим поле $v$ до векторного поля $V$ на некоторую окрестность поверхности $\mathscr{M}$, интегральными кривыми которого служат геодезические многообразия $N$. Обозначим через $F_{t}: N \rightarrow N$ однопараметрическую группу локальных диффеоморфизмов поля $V$, а 
через $\mathscr{M}_{t}=F_{t}(\mathscr{M})$ - соответствуюшую ей вариацию поверхности $\mathscr{M}$. Пусть $S(t)$ - плошадь поверхности $\mathscr{M}_{t}$.

ТеоРема 1. Имеет место формула

$$
\left.\frac{d S}{d t}\right|_{t=0}=-\int_{\mathscr{M}}\langle H(m), v(m)\rangle
$$

u, если средняя кривизна $H(m) \equiv 0$, то

$$
\left.\frac{d^{2} S}{d t^{2}}\right|_{t=0}=\int_{\mathscr{M}}\left\{-\left\|A^{v}\right\|^{2}+\|\nabla v\|^{2}+k(v)\right\},
$$

əдe

$$
\|\nabla v\|^{2}=\sum_{i=1}^{p} \delta_{i}\left|\nabla_{E_{i}} v\right|^{2}
$$

ДоказАТЕЛЬСТво. Обозначим через $\omega(n+1)$-форму объема на многообразии $N$, через $X_{1}, X_{2}, \ldots, X_{p}$ - ортонормированные векторные поля на $\mathscr{M}$, через $v_{1}, v_{2}, \ldots, v_{n-p+1}$ - ортонормированную систему нормальных векторов для поверхности $\mathscr{M}_{t}$. Пусть $F_{t} X_{i}=X_{i}^{*}$. Тогда имеем

$$
S(t)=\int_{\mathscr{M}} \omega\left(X_{1}^{*}, X_{2}^{*}, \ldots, X_{p}^{*}, v_{1}, v_{2}, \ldots, v_{n+1-p}\right) \circ F_{t}(m) .
$$

Для доказательства нам потребуется

Лемма 1. Если $\bar{\nabla}-$ связность в $N$, то $\bar{\nabla} \omega \equiv 0$.

ДокАЗАТЕЛЬСтво. Пусть $\xi_{1}, \xi_{2}, \ldots, \xi_{n+1}$ - ортонормированные векторные поля на $N$. Тогда для любого $Y \in T N$

$$
\begin{aligned}
\left(\bar{\nabla}_{Y} \omega\right)\left(\xi_{1}, \xi_{2}, \ldots, \xi_{n+1}\right) & =\bar{\nabla}_{Y}\left(\omega\left(\xi_{1}, \xi_{2}, \ldots, \xi_{n+1}\right)\right)-\omega\left(\bar{\nabla}_{Y} \xi_{1}, \xi_{2}, \ldots, \xi_{n+1}\right) \\
& -\omega\left(\xi_{1}, \bar{\nabla}_{Y} \xi_{2}, \ldots, \xi_{n+1}\right)-\ldots-\omega\left(\xi_{1}, \xi_{2}, \ldots, \xi_{n}, \bar{\nabla}_{Y} \xi_{n+1}\right) .
\end{aligned}
$$

Ясно, что $\omega\left(\xi_{1}, \xi_{2}, \ldots, \xi_{n+1}\right) \equiv 1, \mathrm{a} \bar{\nabla}_{Y} \xi_{i}$ ортогонален $\xi_{i}$ и, следовательно, линейно зависит от $\xi_{1}, \xi_{2}, \ldots, \widehat{\xi}_{i}, \ldots, \xi_{n+1}$. Здесь $\widehat{\xi}_{i}$ означает, что символ $\xi_{i}$ пропушен. Из этого заключаем, что $\bar{\nabla}_{Y} \omega\left(\xi_{1}, \xi_{2}, \ldots, \xi_{n+1}\right)=0$. Далее, из линейности $\bar{\nabla} \omega$ и того, что $\bar{\nabla}_{Y} \omega$ тензор, заключаем, что $\bar{\nabla} \omega \equiv 0$.

Продолжим доказательство теоремы 1. По определению производной Ли [7]:

$$
\begin{aligned}
\frac{d}{d t}\{ & \left.\omega\left(X_{1}^{*}, X_{2}^{*}, \ldots, X_{p}^{*}, v_{1}, v_{2}, \ldots, v_{n+1-p}\right) \circ F_{t}\right\} \\
& =L_{V}\left\{\omega\left(X_{1}^{*}, X_{2}^{*}, \ldots, X_{p}^{*}, v_{1}, v_{2}, \ldots, v_{n+1-p}\right) \circ F_{t}\right\} \\
& =\bar{\nabla}_{V} \omega\left(X_{1}^{*}, X_{2}^{*}, \ldots, X_{p}^{*}, v_{1}, v_{2}, \ldots, v_{n+1-p}\right) \circ F_{t} .
\end{aligned}
$$

Поэтому

$$
S^{\prime}(t)=\int_{\mathscr{M}} \bar{\nabla}_{V}\left\{\omega\left(X_{1}^{*}, X_{2}^{*}, \ldots, X_{p}^{*}, v_{1}, v_{2}, \ldots, v_{n+1-p}\right)\right\} \circ F_{t} .
$$


В силу леммы 1

$$
\begin{aligned}
S^{\prime}(t) & =\sum_{i=1}^{p} \int_{\mathcal{M}} \omega\left(X_{1}^{*}, \ldots, \bar{\nabla}_{V} X_{i}^{*}, \ldots, X_{p}^{*}, v_{1}, v_{2}, \ldots, v_{n+1-p}\right) \circ F_{t} \\
& =\sum_{j=1}^{n+1-p} \int_{\mathscr{M}} \omega\left(X_{1}^{*}, X_{2}^{*}, \ldots, X_{p}^{*}, v_{1}, \ldots, \bar{\nabla}_{V} v_{j}, \ldots, v_{n+1-p}\right) \circ F_{t} .
\end{aligned}
$$

По построению полей $X_{i}^{*}$ имеем $L_{V} X_{i}^{*}=\bar{\nabla}_{V} X_{i}^{*}-\bar{\nabla}_{X_{i}^{*}} V=0$, а $\bar{\nabla}_{V} v_{j}$ линейно зависим от $X_{1}^{*}, \ldots, X_{p}^{*}, v_{1}, \ldots, \widehat{v}_{j}, \ldots, v_{n+1-p}$. Поэтому из (2)

$$
S^{\prime}(t)=\sum_{i=1}^{p} \int_{\mathscr{M}} \omega\left(X_{1}^{*}, \ldots, \bar{\nabla}_{X_{i}^{*}} V, \ldots, X_{p}^{*}, v_{1}, \ldots, v_{n+1-p}\right) \circ F_{t} .
$$

Дифференцируя еше раз, аналогично получаем

$$
\begin{aligned}
S^{\prime \prime}(t)= & \sum_{i=1}^{p} \int_{\mathscr{M}} \omega\left(X_{1}^{*}, \ldots, \bar{\nabla}_{V} \bar{\nabla}_{X_{i}^{*}} V, \ldots, X_{p}^{*}, v_{1}, \ldots, v_{n+1-p}\right) \circ F_{t} \\
& +\sum_{i \neq j} \omega\left(X_{1}^{*}, \ldots, \bar{\nabla}_{X_{j}^{*}} V, \ldots, \bar{\nabla}_{X_{i}^{*}} V, \ldots, X_{p}^{*}, v_{1}, \ldots, v_{n+1-p}\right) \circ F_{t} \\
& +\sum_{j=1}^{n+1-p} \sum_{i=1}^{p} \int_{\mathscr{M}} \omega\left(X_{1}^{*}, \bar{\nabla}_{X_{i}^{*}} V, \ldots, X_{p}^{*}, v_{1}, \ldots, \bar{\nabla}_{V} v_{j}, \ldots, v_{n+1-p}\right) \circ F_{t} .
\end{aligned}
$$

Далее, учитывая равенства

$$
\begin{aligned}
& \left.\omega\left(X_{1}^{*}, \ldots, \bar{\nabla}_{X_{i}^{*}} V, \ldots, X_{p}^{*}, v_{1}, \ldots, \bar{\nabla}_{V} v_{j}, \ldots, v_{n+1-p}\right)\right|_{\mathscr{M}} \\
& =\omega\left(X_{1}, \ldots, \bar{\nabla}_{X_{i}} v, \ldots, X_{p}, v_{1}, \ldots, \bar{\nabla}_{V} v_{j}, \ldots, v_{n+1-p}\right) \\
& =\omega\left(X_{1}, \ldots, \nabla_{X_{i}} v, \ldots, X_{p}, v_{1}, \ldots, \bar{\nabla}_{V} v_{j}, \ldots, v_{n+1-p}\right) \\
& -\omega\left(X_{1}, \ldots, A^{v}\left(X_{i}\right), \ldots, X_{p}, v_{1}, \ldots, \bar{\nabla}_{V} v_{j}, \ldots, v_{n+1-p}\right) \\
& =\omega\left(X_{1}, \ldots, \nabla_{X_{i}} v, \ldots, X_{p}, v_{1}, \ldots,\left\langle\bar{\nabla}_{V} v_{j}, X_{i}\right\rangle X_{i}, \ldots, v_{n+1-p}\right) \delta_{i} \\
& -\omega\left(X_{1}, \ldots, A^{v}\left(X_{i}\right), \ldots, X_{p}, v_{1}, \ldots,\left\langle\bar{\nabla}_{V} v_{j}, X_{i}\right\rangle X_{i}, \ldots, v_{n+1-p}\right) \\
& =\omega\left(X_{1}, \ldots,\left\langle\nabla_{X_{i}} v, v_{j}\right\rangle v_{j}, \ldots, X_{p}, v_{1}, \ldots,\left\langle\bar{\nabla}_{V} v_{j}, X_{i}\right\rangle X_{i}, \ldots, v_{n+1-p}\right) \delta_{i} \\
& =\omega\left(X_{1}, \ldots, v_{j}, \ldots, X_{p}, v_{1}, \ldots, X_{i}, \ldots, v_{n+1-p}\right)\left\langle\nabla_{X_{i}} V, v_{j}\right\rangle\left\langle\bar{\nabla}_{V} v_{j}, X_{i}\right\rangle \delta_{i} \varepsilon_{j} \\
& =\left\langle\nabla_{X_{i}} V, v_{j}\right\rangle^{2} \omega\left(X_{1}, \ldots, X_{i}, \ldots, X_{p}, v_{1}, \ldots, v_{j}, \ldots, v_{n+1-p}\right) \delta_{i} \varepsilon_{j}, \\
& \text { где } \delta_{i}=\left|X_{i}\right|^{2}, \varepsilon_{j}=\left|v_{j}\right|^{2} \text {; }
\end{aligned}
$$

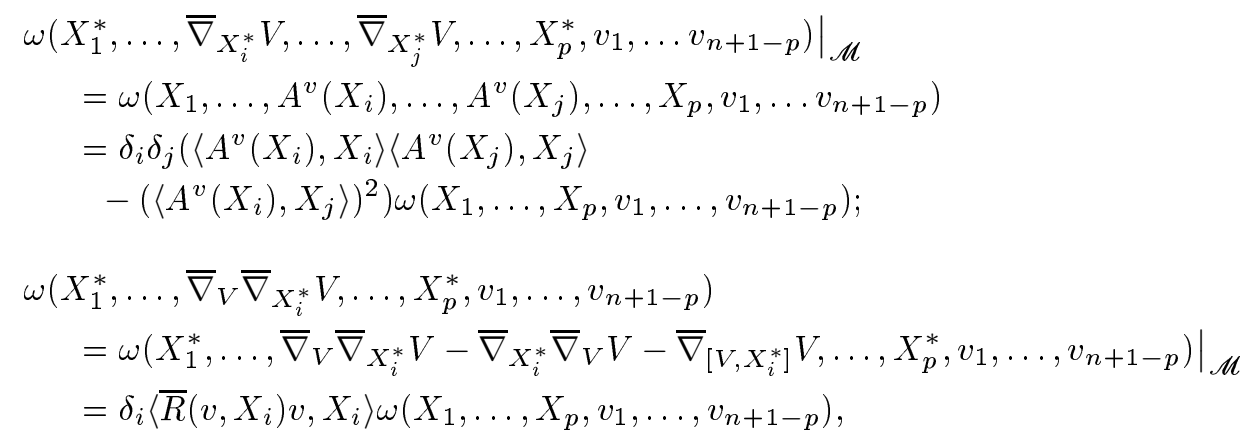


получаем

$$
\begin{aligned}
S^{\prime \prime}(t)=\int_{\mathscr{M}}\left\{\sum_{i=1}^{p} \delta_{i}\left\langle\bar{R}\left(v, X_{i}\right) v, X_{i}\right\rangle\right. & \\
& +\sum_{i \neq j} \delta_{i} \delta_{j}\left(\left\langle A^{v}\left(X_{i}\right), X_{i}\right\rangle\left\langle A^{v}\left(X_{j}\right), X_{j}\right\rangle\right. \\
& \left.\left.-\left(\left\langle A^{v}\left(X_{i}\right), X_{j}\right\rangle\right)^{2}\right)+\sum_{j=1}^{n+1-p} \sum_{i=1}^{p}\left(\left\langle\nabla_{X_{i}} v, v_{j}\right\rangle\right) \varepsilon_{j} \delta_{i}\right\} .
\end{aligned}
$$

Используя, что $H(m) \equiv 0$, находим

$$
\begin{aligned}
\sum_{i \neq j} \delta_{i} \delta_{j}\left(\left\langle A^{v}\left(X_{i}\right), X_{i}\right\rangle\left\langle A^{v}\left(X_{j}\right), X_{j}\right\rangle-\left(\left\langle A^{v}\left(X_{i}\right), X_{j}\right\rangle\right)^{2}\right) & \\
& =\frac{1}{p^{2}}(\langle H, v\rangle)^{2}-\left\|A^{v}\right\|^{2}=-\left\|A^{v}\right\|^{2}
\end{aligned}
$$

С другой стороны, очевидно

$$
\sum_{j=1}^{n+1-p} \sum_{i=1}^{p} \varepsilon_{j} \delta_{i}\left(\left\langle\nabla_{X_{i}} v, v_{j}\right\rangle\right)^{2}=\|\nabla v\|^{2}
$$

Поэтому окончательно

$$
S^{\prime \prime}(0)=\int_{\mathscr{M}}\left\{-\left\|A^{v}\right\|^{2}+\|\nabla v\|^{2}+k(v)\right\} .
$$

Непосредственно из (3) получаем

$$
S^{\prime}(0)=-\int_{\mathscr{M}}\langle H, v\rangle .
$$

Таким образом, теорема 1 доказана полностью.

\section{2. Вариации пространственноподобных поверхностей}

На основании доказанной теоремы для поверхностей с нулевым вектором средней кривизны выполнено $S^{\prime}(0)=0$ и определена форма

$$
Q(v)=\int_{\mathscr{M}}\left\{-\left\|A^{v}\right\|^{2}+\|\nabla v\|^{2}+k(v)\right\} .
$$

Пусть здесь $\mathscr{M}$ пространственноподобна. Естественно выделяются два случая.

Первый случай. Пусть $v=h \xi$, где $\xi$ - некоторое гладкое, единичное, времениподобное сечение нормального расслоения $\mathscr{M}$ и $h$ - функция класса $C^{1}(\mathscr{M})$. Имеем

$$
\begin{gathered}
\|\nabla v\|^{2}=|\nabla h|^{2}+h^{2}|\nabla \xi|^{2}, \\
\left\|A^{v}\right\|^{2}=h^{2}\left\|A^{\xi}\right\|^{2} .
\end{gathered}
$$


Форма (4) переписьвается тогда следующим образом

$$
Q^{\xi}(h)=-\int_{\mathscr{M}}\left\{|\nabla h|^{2}-h^{2}\left(\|\nabla \xi\|^{2}-\left\|A^{\xi}\right\|^{2}+k(\xi)\right)\right\} .
$$

Зафиксируем точку $m_{0} \in M$ и окрестность $\mathscr{U} \subset M$ точки $m_{0}$. Положим

$$
c=\sup _{\mathscr{U}}\left(\|\nabla \xi\|^{2}-\left\|A^{\xi}\right\|^{2}+k(\xi)\right)
$$

Для произвольной $C^{1}$-гладкой функции $h$ с компактным носителем supp $h \subset \mathscr{U}$ в силу (5) имеем

$$
Q^{\xi}(h) \leqslant-\int_{\mathscr{U}}\left\{|\nabla h|^{2}-c h^{2}\right\} \leqslant-\int_{\mathscr{U}}(\lambda-c) h^{2},
$$

где

$$
\lambda=\lambda(\mathscr{U})=\inf _{\varphi \in C_{0}^{1}(\mathscr{U})}\left(\int_{\mathscr{U}}|\nabla \varphi|^{2}\right)\left(\int_{\mathscr{U}} \varphi^{2}\right)^{-1}
$$

- основная частота множества $\mathscr{U}[8]$.

Так как $\lambda(\mathscr{U}) \rightarrow \infty$ при $\operatorname{diam} \mathscr{U} \rightarrow 0$, то для достаточно малой окрестности $\mathscr{U}$ точки $m_{0}$ величина $Q^{\xi}(h) \leqslant 0$. Тем самьм вариация поверхности $\mathscr{M}$ в направлении векторного поля $\xi$ не увеличивает площади, и поверхность $\mathscr{M}$ является локально максимальной в направлении векторного поля $\xi$.

Второй случай. Пусть $v=h \eta$, где $\eta$ - некоторое гладкое, единичное пространственноподобное сечение нормального расслоения поверхности $\mathscr{M}$ и $h \in C^{1}(\mathscr{M})$. Поскольку $\mathscr{M}$ пространственноподобна, то данный случай реализуется лишь при codim > 1. Формула второй вариации (4) переписывается здесь в виде

$$
Q^{\eta}(h)=\int_{\mathscr{M}}\left\{|\nabla h|^{2}-h^{2}\left(-\|\nabla \eta\|^{2}+\left\|A^{\eta}\right\|^{2}-k(\eta)\right)\right\} .
$$

Если носитель функции $h$ достаточно мал, то, как и выше, выводим $Q^{\eta}(h) \geqslant 0$. Следовательно, вариация поверхности $\mathscr{M}$ в направлении векторного поля $\eta$ не уменьшает площади, и поверхность М является локально минимальной в этом направлении.

\section{3. Вариации времениподобных поверхностей}

Перейдем к рассмотрению случая времениподобных поверхностей нулевой средней кривизны. Однако в данном случае мы ограничимся более узким классом поверхностей. Дадим точные определения. Пусть $H-n$-мерное, связное, риманово многообразие класса $C^{2}$ с элементом длины $d s_{H}$ и пусть $\delta(x)$ - положительная $C^{1}$-функция на $H$. Лоренцево многообразие $N=H \times \mathbb{R}$ с метрикой

$$
d s^{2}=-\delta(x) d t^{2}+d s_{H}^{2}
$$

будем называть искривленным лоренцевым произведением и обозначать через $N=H \times_{\delta} \widehat{\mathbb{R}}$. Здесь $\widehat{\mathbb{R}}-$ вешественная прямая, снабженная отрицательно определенной метрикой $\left(-d t^{2}\right)$. Введем обозначение $H_{\tau}=\left\{(x, t) \in H \times_{\delta} \widehat{\mathbb{R}}: t=\tau\right\}$. 
Рассмотрим поверхность $\mathscr{M}=(M, u)$, погруженную в $H \times_{\delta} \widehat{\mathbb{R}}$. Будем говорить, что поверхность $\mathscr{M}$ есть лента с проекиией $(a, b)$, где $-\infty \leqslant a<b \leqslant+\infty$, если

1. для всякого $\tau \in(a, b)$ множество

$$
E_{t}=u(\partial M) \cap H_{\tau} \neq \varnothing ;
$$

2. для всяких $\tau_{1}, \tau_{2} \in(a, b), \tau_{1}<\tau_{2}$, множества

$$
M\left(t_{1}, t_{2}\right)=u(M) \cap\left\{(x, t) \in H \times_{\delta} \widehat{\mathbb{R}}: a<\tau_{1}<t<\tau_{2}<b\right\}
$$

предкомпактны;

3. если точка $m \in \partial M$ есть точка гладкости края $\partial M$ и $\nu$ - внешняя нормаль на $M$ к $\partial M$, то $\langle e, \nu\rangle=0$, где $e-$ векторное поле в $H \times_{\delta} \widehat{\mathbb{R}}$, определяемое соотношением: $\bar{\nabla}_{e} h(x, t)=\frac{\partial}{\partial t} h(x, t)$ для всех $h \in C^{1}(\mathscr{M})$;

4. если $m \in \partial M$ - точка нерегулярности края $\partial M$, то контингенция cont $_{u(m)} u(M)$ не содержит световых лучей.

Конечную или бесконечную разность $b-a$ будем назьвать протяженностью ленты. Если $a=-\infty, b=+\infty$, то $\mathscr{M}$ называется лентой в целом.

Поверхность $\mathscr{M}=(M, u)$ в $N$ назьвается поверхностью трубчатого типа $c$ проекиией $(a, b)$, если $M$ - многообразие без края и $(M, u)$ обладает вторым из вьшеперечисленных свойств.

Примеры трубок и лент в искривленных лоренцевых произведениях можно найти в [9]-[12].

Аналогичньм образом данные понятия вводятся и для поверхностей в искривленных римановых произведениях [5].

Пусть $\mathscr{M}=(M, u)$ - времениподобная лента с нулевым вектором средней кривизны в $H \times_{\delta} \widehat{\mathbb{R}}$. Среди всех вариаций ленты мы выделяем два класса вариаций. Во-первых, это вариации, определяемые $C^{1}$-гладкими функциями $h$ такими, что $|\nabla h|^{2} \leqslant 0$ и, во-вторых, $-C^{1}$-вариации, для которых $|\nabla h|^{2} \geqslant 0$.

Пусть $v=h \cdot \eta$, где $\eta$ - единичное, пространственноподобное сечение нормального расслоения поверхности $\mathscr{M}, h \in C^{1}(\mathscr{M})$. Тогда форма (4) перепишется в виде

$$
Q^{\eta}(h)=\int_{\mathscr{M}}\left\{|\nabla h|^{2}-h^{2}\left(\left\|A^{\eta}\right\|^{2}-\|\nabla \eta\|^{2}-k(\eta)\right)\right\} .
$$

Пусть

$$
c=\sup _{M\left(t_{1}, t_{2}\right)} \frac{\left\|A^{\eta}\right\|^{2}-\|\nabla \eta\|^{2}-k(\eta)}{-|\nabla f|^{2}} .
$$

Положим $h(m)=h(f(m))$. Тогда

$$
Q^{\eta}(h) \leqslant \int_{\mathscr{M}}\left\{|\nabla h|^{2}+c h^{2}|\nabla f|^{2}\right\} .
$$

Применяя формулу Кронрода-Федерера, получаем

$$
-I\left(\int_{t_{1}}^{t_{2}} h^{\prime 2}(t)+c h^{2}(t) d t\right)<0,
$$


где

$$
I=\int_{E_{t}}|\nabla f|=\text { const }>0 .
$$

Неравенство (7) вьполнено, если $t_{2}-t_{1}<\pi / \sqrt{-c}$ при $c<0$ в силу неравенства Виртингера, а при $c>0$ оно очевидно. Поэтому ленты с нулевым вектором средней кривизны локально максимальны относительно рассмотренных вариаций.

ЗАмЕчАниЕ 1. Во всех рассмотренных случаях локальная знакоопределенность формы (4) зависит от знака интеграла Дирихле. Однако при глобальном рассмотрении поверхности нулевой средней кривизны второе слагаемое в (4) может оказаться весомее и тогда форма (4) поменяет знак на противоположный. Из этих соображений понятие устойчивости мы определяем как способность формы (4) сохранять знак на множестве всех допустимых вариаций.

ОПРЕДЕЛЕНИЕ. Будем говорить, что поверхность $\mathscr{M}=(M, u)$, погруженная в лоренцево многообразие $N$ с нулевым вектором средней кривизны устойчива относительно вариачий по направлению единичного времениподобного (пространственноподобного) сечения $v$ нормального расслоения, если $Q^{v}(h)<0$ (если $\left.Q^{v}(h)>0\right)$ для всех $h \in C^{1}(\mathscr{M})$ с компактньп носителем.

В случае гиперповерхностей поле нормалей определяется однозначно и мы будем говорить просто, что поверхность устойчива или неустойчива.

ПримеР 1. Рассмотрим поверхность $\mathscr{M}$ в $R_{1}^{4}$, заданную уравнением

$$
x_{0}=t, \quad x_{1}=\sin t \cos \psi, \quad x_{2}=\sin t \sin \psi, \quad x_{3}=0 .
$$

Введем обозначения. Пусть $\eta=e_{3}$, а $\xi$ - вектор единичной нормали, ортогональньй $\eta$. Ясно, что данная пара векторов образует ортонормированный базис нормального пространства к поверхности $\mathscr{M}$. Нетрудно проверить, что для этой поверхности справедливы равенства

$$
\begin{gathered}
H \equiv 0, \\
\left\|A^{\eta}\right\|^{2}=0,\left\|A^{\xi}\right\|^{2}=\frac{2}{\sin ^{4} t}, \\
|\nabla f(m)|^{2}=-\frac{1}{\sin ^{2} t},
\end{gathered}
$$

где $f(m)$ - сужение координатной функции $x_{0}$ на поверхность $\mathscr{M}$.

Действительно, векторы

$$
\begin{aligned}
& X_{0}=(\cos t \cos \psi, \cos t \sin \psi, 0,1), \\
& X_{1}=(-\sin t \sin \psi, \sin t \cos \psi, 0,0)
\end{aligned}
$$

образуют базис в касательной плоскости к поверхности $\mathscr{M}$, причем

$$
\left|X_{0}\right|^{2}=-\left|X_{1}\right|^{2}=-\sin ^{2} t, \quad\left\langle X_{0}, X_{1}\right\rangle=0
$$

Поэтому поверхность времениподобна. Равенство $A^{\eta}=0$ вьполнено в силу того, что нормальное поле $\eta$ постоянно вдоль поверхности $\mathscr{M}$. Для вектора $\xi$ можем записать

$$
\xi=\left(\frac{\cos \psi}{\sin t}, \frac{\sin \psi}{\sin t}, 0, \frac{\cos t}{\sin t}\right),
$$


поэтому по определению гомоморфизма $A^{\xi}$

$$
\begin{aligned}
& A^{\xi}\left(X_{0}\right)=-\bar{\nabla}_{X_{0}} \xi=\left(\frac{\cos t \cos \psi}{\sin ^{2} t}, \frac{\cos t \sin \psi}{\sin ^{2} t}, 0, \frac{1}{\sin ^{2} t}\right), \\
& A^{\xi}\left(X_{1}\right)=\left(-\frac{\sin \psi}{\sin t}, \frac{\cos \psi}{\sin t}, 0,0\right) .
\end{aligned}
$$

Используя (1), (11) и (12) нетрудно подсчитать, что вектор средней кривизны $\mathscr{M}$ равен нулю. Действительно,

$$
\langle H, \eta\rangle=\left\langle A^{\eta}\left(X_{0}\right), X_{0}\right\rangle+\left\langle A^{\eta}\left(X_{1}\right), X_{1}\right\rangle=0
$$

и

$$
\langle H, \xi\rangle=\left\langle A^{\xi}\left(X_{0}\right), X_{0}\right\rangle+\left\langle A^{\xi}\left(X_{1}\right), X_{1}\right\rangle=\frac{\cos ^{2} t-1}{\sin ^{2} t}+1=0 .
$$

Далее, из (11) и (12) получаем

$$
\left\|A^{\xi}\right\|^{2}=\frac{2}{\sin ^{4} t}
$$

Градиент функции времени равен $\nabla f=-e_{0}^{T}$ и из $(9),(10)$ непосредственно следует, что

$$
-|\nabla \psi|^{2}=|\nabla f|^{2}=-\frac{1}{\sin ^{2} t}
$$

Рассмотрим сечение $\nu$ нормального расслоения к $\mathscr{M}$, определяемое по правилу $\nu=\eta \cos k \psi+\xi \sin k \psi$, где $k$ целое. Используя (13), непосредственно проверяется, что $\|\nabla \nu\|^{2}=-k^{2}|\nabla f|^{2}$. Теперь из (8) находим

$$
Q^{\nu}(h)=\int_{\mathscr{M}}\left\{|\nabla h|^{2}-h^{2}\left(\frac{2}{\sin ^{4} t}-\frac{k^{2}}{\sin ^{2} t}\right)\right\} .
$$

Пусть, далее, $h(m)=h(f(m))=h(t)$, причем $h(t)=0$ при $0<t<\pi / 4$ и $3 \pi / 4<t<\pi$. Тогда, используя формулу Кронрода-Федерера, получаем

$Q^{\nu}(h) \geqslant \int_{\mathscr{M}}\left\{|\nabla h|^{2}-h^{2}\left(\frac{1}{\sin ^{2} t}-\frac{k^{2}}{\sin ^{2} t}\right)\right\}=-2 \pi \int_{\pi / 4}^{3 \pi / 4} h^{\prime 2}(t)-h^{2} k^{2}+h^{2}>0$

для функции $h(t)=|\sin 4 t|$ и при $k=5$. Заметим, что вектор $\nu$ получается поворотом вектора $\eta$ на угол $k \psi$ в нормальном пространстве к поверхности $\mathscr{M}$. Поэтому величина $k$ характеризует число поворотов векторного поля $\nu$. Тем самьм можно заключить, что неустойчивость времениподобных трубок нулевой средней кривизны относительно вариаций, определяемых функциями $h(m)$ с времениподобньм градиентом, может иметь место относительно сильно “колеблющихся" сечений нормального расслоения. 
ПримеР 2. Рассмотрим поверхность $\mathscr{M} \subset R_{1}^{3}$, заданную уравнением

$$
x_{0}=\psi, \quad x_{1}=r \cos \psi, \quad x_{2}=r \sin \psi,
$$

где $-1<r<1,0<\psi<\pi$. Метрика этой поверхности имеет вид

$$
d s_{\mathscr{M}}^{2}=d r^{2}-\left(1-r^{2}\right) d \psi^{2} .
$$

Это следует из того, что векторы

$$
X_{0}=(1,-r \sin \psi, r \cos \psi), \quad X_{1}=(0, \cos \psi, \sin \psi)
$$

образуют ортогональный базис в касательном пространстве поверхности $\mathscr{M}$ и единичньй вектор нормали равен

$$
\xi=\left(\frac{r}{\sqrt{1-r^{2}}}, \frac{-\sin \psi}{\sqrt{1-r^{2}}}, \frac{\cos \psi}{\sqrt{1-r^{2}}}\right) .
$$

Имеем

$$
\begin{aligned}
& A\left(X_{0}\right)=-\bar{\nabla}_{X_{0}} \xi=\left(0, \frac{-\cos \psi}{\sqrt{1-r^{2}}}, \frac{-\sin \psi}{\sqrt{1-r^{2}}}\right) \\
& A\left(X_{1}\right)=\left(\left(\frac{r}{\sqrt{1-r^{2}}}\right)^{\prime},-\sin \psi\left(\frac{1}{\sqrt{1-r^{2}}}\right)^{\prime}, \cos \psi\left(\frac{1}{\sqrt{1-r^{2}}}\right)^{\prime}\right) .
\end{aligned}
$$

Используя (14) и (15) проверяем, что средняя кривизна поверхности $\mathscr{M}$ равна нулю и

$$
\|A\|^{2}=\frac{2}{\left(1-r^{2}\right)^{2}}
$$

Если в формуле второй вариации положить $h(m)=h(r)$ с $h(-1)=h(1)=0$, то мы получим

$$
Q(h)=\pi \int_{-1}^{1}\left(h^{\prime 2}-h^{2} \frac{2}{\left(1-r^{2}\right)^{2}}\right) \sqrt{1-r^{2}} d r .
$$

Выбирая здесь $h(r)=\sqrt{1-r^{2}}$, приходим к выводу, что $Q(h)<0$ и, следовательно, поверхность $\mathscr{M}$ относительно рассматриваемых вариаций не является глобально минимальной. Однако для достаточно малых $\delta>0$ для любой функции $h(r)$ такой, что $h(r)=0$ при $|r|>\delta$ величина $Q(h)$ положительно определена. Следовательно, рассматривая нашу поверхность только при $|r|<\delta$ мы получаем глобально минимальную поверхность, хотя, как только что мы убедились, в целом она не является таковой. Заметим, что в случае пространственноподобных гиперповерхностей в плоском пространстве Минковского форма (5) всегда отрицательно определена, а поверхность, соответственно, глобально максимальна. В нашем примере ситуация иная и проблема устойчивости времениподобных поверхностей нулевой средней кривизны относительно вариаций, определяемых функциями с пространтсвенноподобными градиентами, не является тривиальной даже в случае коразмерности 1. 


\section{4. Основные результаты}

На протяжении всей оставшейся части статьи будем считать, что поверхность $\mathscr{M}=(M, u)$ пространственноподобна.

Для формулировки основных результатов работы нам потребуется понятие емкости [13], [14]. Пусть $M$ риманово многообразие с кусочно-гладким краем или без него. Рассмотрим на $M$ два замкнутых непересекающихся подмножества $P$ и $Q$. Величина

$$
\operatorname{cap}(P, Q)=\inf \int_{\mathscr{M}}|\nabla \varphi|^{2},
$$

где точная нижняя грань берется по всем локально липшицевым функциям $\varphi: M \rightarrow \mathbb{R}$ таким, что $\varphi(m)=1$, если $m \in P$, и $\varphi(m)=0$, если $m \in Q$, называется емкостью конденсатора $(P, Q)$.

Многообразие $M$ назьвается параболическим, если существует исчерпание $M$ открытыми множествами

$$
D_{k}, \quad k=1,2, \ldots, \quad D_{1} \Subset D_{2} \Subset \cdots, \quad \bigcup_{k \geqslant 1} D_{k}=M,
$$

такое, что для любого компакта $K \subset M$ выполнено

$$
\liminf _{k \rightarrow+\infty} \operatorname{cap}\left(K, \overline{D_{k} \backslash D_{k-1}}\right)=0 .
$$

Tеорема 2. Пусть $\mathscr{M}=(M, u)$ - гиперповерхность нулевой средней кривизньи в лоренцевом многообразии $N$. Обозначим через $\bar{S}$ и $S$ скалярнье кривизны $N$ и $\mathscr{M}$, соответственно, через $r=r(\xi)$ кривизну Риччи в направлении единичной нормали $\xi к$ поверхности $\mathscr{M}$. Пусть $S-\bar{S}-r<0$ и существуют область $\Omega$ и компакт $P \Subset \Omega$ такие, ито

$$
-\int_{P}(S-\bar{S}-r)>\operatorname{cap}(P, \overline{\partial \Omega \backslash \partial M}) .
$$

Тогда М̆ неустойчива.

Теорема 3. Пусть $\mathscr{M}=(M, u)-(n-1)$-мерная поверхность нулевой средней кривизны в $(n+1)$-мерном лоренцевом многообразии $N$, и $\eta$ - фиксированное единичное пространственноподобное сечение нормального расслоения $\mathscr{M}$, вдоль которого $k(\eta) \leqslant 0$. Тогда если существуют область $\Omega$ и компакт $P \Subset \Omega$ такие, что

$$
\int_{P}\left\|A^{\eta}\right\|^{2}>\operatorname{cap}(P, \overline{\partial \Omega \backslash \partial M})
$$

то $\mathscr{M}$ неустойчива относительно $\eta$.

Из теоремы 3 получается следуюшее 
СЛЕДСТВИЕ 1. Пусть $\mathscr{M}=(M, u)-$ минимальная гиперповерхность в римановом многообразии $\bar{M}$ неотричательной кривизны Риччи $r=r(n) \geqslant 0$ по направлению единичной нормали $n$ к поверхности $\mathscr{M}$. Пусть $\bar{S}$ и $S$ обозначают скалярные кривизны $\bar{M}$ и $\mathscr{M}$, соответственно. Если существуют область $\Omega$ и компакт $P \Subset \Omega$ такие, что

$$
\int_{P}(\bar{S}-S-r)>\operatorname{cap}(P, \overline{\partial \Omega \backslash \partial M})
$$

то $\mathscr{M}$ неустойчива.

Данное утверждение проиллюстрируем на следующем примере. В этом примере емкость конденсаторов специального вида точно вычисляется через внешние характеристики поверхности.

ПримеР 3. Рассмотрим цилиндр $C=S^{n-1} \times \mathbb{R} \subset \mathbb{R}^{n+1}$. Тогда элемент длины $C$ может быть представлен в виде

$$
d s_{C}^{2}=d t^{2}+d r^{2}+\sin ^{2} r d l^{2},
$$

где $d l$ и $r$, соответственно, - элемент длины и геодезическое расстояние от некоторой фиксированной точки единичной $(n-2)$-мерной сферы.

Лемма 2. Существует радиально-симметричная, периодическая по $t$, минимальная, трубчатая в целом поверхность в $C$.

ДокАЗАТЕЛЬСтво. Пусть $x \in S^{n-1}, \theta \in S^{n-2}$. Будем искать соответствующую поверхность в виде

$$
t=\tau, \quad x=r(\tau) \theta .
$$

Непосредственные вычисления, аналогичные проведенным в [9], показывают, что таким образом заданная поверхность будет минимальной в $C$, если $r(\tau)$ удовлетворяет дифференциальному уравнению

$$
r^{\prime 2}(\tau)=c^{2} \sin ^{2(n-2)} r(\tau)-1
$$

для некоторой постоянной $0<c<1$. Это несложно проверить, замечая, что в метрике

$$
d r^{2}+\sin ^{2} r d l^{2}
$$

единичной $(n-1)$-мерной сферы имеет место соотношение

$$
\Delta r=(n-2) \operatorname{ctg} r
$$

Положим

$$
t_{n, c}=\int_{r_{0}}^{\pi} \frac{c d r}{\sqrt{\sin ^{2(n-2)} r-c^{2}}}, \text { где } c=\sin ^{n-2} r_{0} .
$$

На интервале $\left(r_{0}, 2 \pi-r_{0}\right)$ функция

$$
\tau(r)=\int_{r_{0}}^{r} \frac{c d r}{\sqrt{\sin ^{2(n-2)} r-c^{2}}}
$$


монотонно возрастает, и поэтому мы можем рассматривать на интервале $\left(-t_{n, c}, t_{n, c}\right)$ функцию $r(\tau)$, обратную к $\tau(r)$. Эта функция может быть симметрично $C^{2}$-гладко продолжена на всю числовую прямую в силу того, что $r^{\prime}\left(-t_{n, c}\right)=0$ и $r^{\prime}\left(t_{n, c}\right)=0$. Лемма доказана.

Элемент длины поверхности (20) имеет вид

$$
d s^{2}=c^{2} \sin ^{2(n-2)} r(\tau) d \tau^{2}+\sin ^{2} r(t) d l^{2} .
$$

Выберем функцию $h(x, \tau)=h(\tau)=\left(T_{1}-|\tau|\right) /\left(T_{1}-T_{0}\right)$ при $T_{0}<|\tau|<T_{1}, h(\tau)=0$ при $|\tau| \geqslant T_{1}$ и $h(\tau)=1$ при $|\tau| \leqslant T_{0}$. Для формы $Q(h)$ тогда получим

$$
Q(h) \leqslant \frac{2}{\left(T_{1}-T_{0}\right)^{2}} \int_{T_{0}}^{T_{1}}|\nabla t|^{2} \sin ^{2(n-2)} r(\tau) \int_{S^{n-2}} d l d \tau-\int_{-T_{0}}^{T_{1}} \int_{S^{n-2}}\|A\|^{2} d l d \tau .
$$

Для первого интеграла имеем

$$
\int_{T_{0}}^{T_{1}}|\nabla t|^{2} \sin ^{2(n-2)} r(\tau) \int_{S^{n-2}} d l d \tau=c^{-2} \omega_{n-2}\left(T_{1}-T_{0}\right),
$$

где $\omega_{n-2}-$ площадь единичной $(n-2)$-мерной сферы $S^{n-2}$. Первое слагаемое в $(21)$ убывает и стремится к нулю при $T_{1} \rightarrow \infty$. Поэтому подходящим выбором $T_{1}>T_{0}$ можно добиться того, чтобы $Q(h)<0$, что означает неустойчивость $\mathscr{M}$.

Пусть $\mathscr{M}=(M, u)$ - трубка или лента с нулевым вектором средней кривизны коразмерности 2 в $H \times{ }_{\delta} \widehat{\mathbb{R}}$. Через $\eta$ обозначим сечение нормального расслоения поверхности $\mathscr{M}$ такое, что $\langle\eta, e\rangle=0,|\eta|^{2}=1$. Здесь векторное поле $е$ описано в п. 3 определения лент. Вариации поверхности $\mathscr{M}$ по направлению такого поля будем называть горизонтальнымми.

Поверхность $\mathscr{M}=(M, u)$ будем называть горизонтально вполне геодезическим подмногообразием в $H \times{ }_{\delta} \widehat{\mathbb{R}}$, если $\left\|A^{\eta}\right\|^{2} \equiv 0$ на $\mathscr{M}$. Далее, для поверхностей в $H \times{ }_{\delta} \widehat{\mathbb{R}}$ определим характеристику роста относительно функции $\delta(x)$. Именно, положим

$$
\delta_{1}(\tau)=\min _{u(M) \cap H_{\tau}} \delta(x)
$$

Имеет место

ТЕОРема 4. Пусть $\mathscr{M}=(M, u)$ - лента или трубка в целом нулевой средней кривизнь, погруженная в искривленное лоренчево произведение $H \times_{\delta} \widehat{\mathbb{R}}$ и $Н$ - риманово многообразие неотрицательной секчионной кривизны. Предположим, что $\sqrt{\delta(x)}$ выпукла на $H$ и

$$
\liminf _{t \rightarrow+\infty} \frac{1}{t^{2}} \int_{-t}^{t} \frac{d \tau}{\delta_{1}(\tau)}=0
$$

Тогда если М̆ устойчива относительно горизонтальных вариаций, то она является горизонтально вполне геодезическим подмногообразием.

СЛЕДСТВИЕ 2. Пусть $\mathscr{M}=(M, u)-(n-1)$-мерная устойчивая относительно горизонтальных вариаций лента в иелом в пространстве Минковского $R_{1}^{n+1}$. Тогда $\mathscr{M}$ лежсит во времениподобной гиперплоскости. 
СЛЕДСТВИЕ 3. Пусть $\mathscr{M}-n$-мерная устойчивая минимальная трубка или лента в целом в искривленном римановом произведении $H \times_{\delta} \mathbb{R}$. Предположим, что кривизна Риччи многообразия $H$ неотрицательна и $\sqrt{\delta(x)}$ выпукла на Н. Если

$$
\liminf _{t \rightarrow+\infty} \frac{1}{t^{2}} \int_{-t}^{t} \frac{d \tau}{\delta_{1}(\tau)}=0
$$

то $\mathscr{M}$ - вполне геодезическое подмногообразие.

Поскольку в евклидовом пространстве вполне геодезические подмногообразия исчерпываются плоскостями, то мы получаем следуюший вариант теоремы до Кармо и Пенга [15], анонсированный в [5].

СлЕДСТвИЕ 4. Всякая устойчивая минимальная лента в целом является частью плоскости.

Пусть $\mathscr{M}=(M, u)$ - максимальная односвязная лента в $R_{1}^{3}$. Рассмотрим на $\mathscr{M}$ функцию $\varphi(m)$, определенную следуюшим образом. Если точка $u(m)$ лежит на сечении $E_{t}$ поверхности $\mathscr{M}$ гиперплоскостью ортогональной оси времени, то $\varphi(m)$ есть значение угла между касательной к $E_{t}$ и направлением, определяемым некоторым фиксированным вектором $e$. В силу односвязности $\mathscr{M}$ функция $\varphi(m)$ корректно определена. Так как гауссово отображение максимальной поверхности в $R_{1}^{3}$ голоморфно, нетрудно показать, что функция $\varphi(m)$ гармонична в метрике поверхности $\mathscr{M}$. Следующая теорема, анонсированная в [16], выделяет геликоид среди всех неустойчивых лент в целом.

Теорема 5. Пусть $\mathscr{M}=(M, u)-$ максимальная лента в иелом в $R_{1}^{3}, u$ функиия $\varphi(m)$ удовлетворяет условию

$$
\limsup _{t \rightarrow+\infty} \frac{\max _{E_{t}}|\varphi(m)|}{t}<+\infty
$$

Тогда $\mathscr{M}-$ часть геликоида.

\section{5. Доказательство теоремы 2}

В качестве первого шага докажем вспомогательную лемму, которой неоднократно будем пользоваться.

Лемма 3. Пусть на римановом многообразии $M$ задана не равная тождественно нулю неотрицательная функция $F(m)$. Тогда для любой ограниченной области $\Omega$ и компакта $P \Subset \Omega$ существует $C^{1}$-гладкая функиия $h_{0}$, $h_{0}=0$ на $\partial \Omega \backslash \partial M, \partial л я$ которой

$$
Q_{F}\left(h_{0}\right)=\int_{M}\left\{\left|\nabla h_{0}\right|^{2}-F h_{0}^{2}\right\} \leqslant \operatorname{cap}(P, \overline{\partial \Omega \backslash \partial M})-\int_{P} F
$$

В частности, если $M$ имеет параболический тип, то $Q_{F}\left(h_{0}\right)<0$ для некоmорой $h_{0}$. 
ДокаЗАТЕЛЬСтво. Рассмотрим краевую задачу

$$
\begin{aligned}
\Delta h(m) & =0, & & m \in \Omega, \\
h(m) & =0, & & m \in \overline{\partial \Omega \backslash \partial M}, \\
h(m) & =1, & & m \in P .
\end{aligned}
$$

Известно [13], что эта задача имеет решение $h_{0}(m)$, причем

$$
\operatorname{cap}(P, \overline{\partial \Omega \backslash \partial M})=\int_{M}\left|\nabla h_{0}\right|^{2} .
$$

Тогда, учитьвая, что $h_{0}=1$ на $P$, получаем

$$
Q_{F}\left(h_{0}\right)=\operatorname{cap}(P, \overline{\partial \Omega \backslash \partial M})-\int_{M} F h_{0}^{2} \leqslant \operatorname{cap}(P, \overline{\partial \Omega \backslash \partial M})-\int_{M} F .
$$

Лемма доказана.

ДОКАЗАТЕЛЬСТво ТЕОРЕМЫ 2. Прежде всего преобразуем выражение

$$
-\left\|A^{\xi}\right\|^{2}+\|\nabla \xi\|^{2}+k(\xi)
$$

Поскольку $\mathscr{M}$ гиперповерхность, то $\nabla \xi \equiv 0[17]$, а

$$
k(\xi)=\sum_{i=1}^{n}\left\langle\bar{R}\left(E_{i}, \xi\right) E_{i}, \xi\right\rangle=-r(\xi)=-r
$$

где $r(\xi)$ - кривизна Риччи многообразия $N$ по направлению $\xi$. Далее, $\left\|A^{\xi}\right\|^{2}=$ $\|B\|^{2}=\sum_{i, j}\left|B\left(E_{i}, E_{j}\right)\right|^{2}$ - норма второй фундаментальной формы поверхности $\mathscr{M},\left\{E_{i}\right\}_{i=1}^{n}$ - базис касательного пространства к $\mathscr{M}$. Полагая $E_{n+1}=\xi$ и учитывая, что $H \equiv 0$, получаем

$$
\begin{aligned}
\|B\|^{2} & =\sum_{i, j=1}^{n}\left|B\left(E_{i}, E_{j}\right)\right|^{2}=\sum_{i, j=1}^{n}\left(\left\langle B\left(E_{i}, E_{j}\right), B\left(E_{i}, E_{j}\right)\right\rangle-\left\langle B\left(E_{i}, E_{i}\right), B\left(E_{j}, E_{j}\right)\right\rangle\right) \\
& =\sum_{i, j=1}^{n}\left(\left\langle R\left(E_{i}, E_{j}\right) E_{i}, E_{j}\right\rangle-\left\langle\bar{R}\left(E_{i}, E_{j}\right) E_{i}, E_{j}\right\rangle\right) \\
& =S+\sum_{i=1}^{n} \sum_{j=1}^{n}\left\langle\bar{R}\left(E_{i}, E_{j}\right) E_{i}, E_{j}\right\rangle \\
& =S+\sum_{i=1}^{n} \sum_{j=1}^{n+1}\left\langle\bar{R}\left(E_{i}, E_{j}\right) E_{i}, E_{j}\right\rangle\left|E_{j}\right|^{2} \\
& =\sum_{i=1}^{n}\left\langle\bar{R}\left(E_{i}, E_{n+1}\right) E_{i}, E_{n+1}\right\rangle .
\end{aligned}
$$

Здесь мы воспользовались уравнениями Гаусса [18, с. 59], где $R($, ) обозначает тензор кривизны $\mathscr{M}$. Теперь из $[6$, с. 360,361$]$ получаем

$$
\|B\|^{2}=S-\bar{S}-2 r(\xi) .
$$


Подставляя найденные величины в (5), используя лемму 3 и условие (17), приходим к требуемому.

Следуюший пример показывает, что класс поверхностей, рассматриваемый в теореме 2 не пуст. Пусть $S_{1}^{n}$ - пространство де-Ситтера 1-го рода [6]:

$$
S_{1}^{n}=\left\{x \in R_{1}^{n+2}:-x_{1}^{2}+x_{2}^{2}+\cdots+x_{n+2}^{2}=1\right\},
$$

где $R_{1}^{n+2}$ - $(n+2)$-мерное пространство Минковского. Поверхность $\mathscr{M}$ в $S_{1}^{n}$, заданная уравнением $x_{1}=0$, является вполне геодезическим подмногообразием и, следовательно, $H \equiv 0$. Непосредственные вычисления дают, что $S-\bar{S}-r=-n<0$. А поскольку поверхности вида $\mathscr{M}_{\varepsilon}=\left\{x_{1}=\varepsilon\right\}$ имеют площадь бо́льшую, чем плошадь $\mathscr{M}$, то $\mathscr{M}$ неустойчива.

\section{6. Доказательство теоремы 3}

Заметим, что если поверхность $\mathscr{M}$ коразмерности 2 , а $\eta$ - единичное пространственноподобное сечение нормального расслоения, то $\|\nabla \eta\|^{2} \leqslant 0$. Поэтому из (6)

$$
Q^{\eta}(h) \leqslant \int_{\mathscr{M}}\left\{|\nabla h|^{2}-\left(\left\|A^{\eta}\right\|^{2}-k(\eta)\right) h^{2}\right\} .
$$

В силу того, что $k(\eta) \leqslant 0$, имеем

$$
Q^{\eta}(h) \leqslant \int_{\mathscr{M}}\left\{|\nabla h|^{2}-h^{2}\left\|A^{\eta}\right\|^{2}\right\} .
$$

Полагая $F(m)=\left\|A^{\eta}\right\|^{2}$, из леммы 3 и (18) получаем требуемое.

Для доказательства следствия 1 достаточно заметить, что $u: M \rightarrow \bar{M} \subset N=$ $\bar{M} \times \widehat{\mathbb{R}}$ - погружение с нулевым вектором средней кривизны, из (24) следует $\left\|A^{\eta}\right\|^{2}=$ $\bar{S}-S-r$ и (19) эквивалентно (18).

СЛЕДСТВИЕ 5. Пусть $\mathscr{M}$ - минимальная гиперповерхность в $\mathbb{R}^{n+1}, B(r)$ геодезический шар радиуса $r$ на $\mathscr{M}$ с иентром в некоторой точке $m_{0} \in \mathscr{M}$, $l(r)-(n-1)$-мерная мера Хаусдорфа граничь $\partial B(r), S$ - скалярная кривизна поверхности М. Тогда, если существуют $0<\rho_{0}<\rho$ такие, что

$$
\begin{aligned}
& \int_{B\left(\rho_{0}\right)}|S|>\frac{(n-2) l(\rho)}{\rho^{n-1}} \cdot \frac{\left(\rho / \rho_{0}\right)^{n-2}}{\left(\rho / \rho_{0}\right)^{n-2}-1} \quad \text { npu } \quad n>2, \\
& \int_{B\left(\rho_{0}\right)}|S|>\frac{l(\rho)}{\rho \log \left(\rho / \rho_{0}\right)} \quad \text { npu } n=2
\end{aligned}
$$

то $\mathscr{M}$ неустойчива.

ДокАЗАТЕЛЬСтво. Действительно, полагая в (16)

$$
\varphi(m)=\left(\int_{r}^{\rho} \frac{d \tau}{l(\tau)}\right)\left(\int_{\rho_{0}}^{\rho} \frac{d \tau}{l(\tau)}\right)^{-1}
$$


получим

$$
\begin{aligned}
\operatorname{cap}\left(B\left(\rho_{0}\right), \partial B(\rho)\right) \leqslant \int_{B(\rho)}|\nabla \varphi|^{2} & =\left(\int_{\rho_{0}}^{\rho} \frac{d \tau}{l(\tau)}\right)^{-2} \int_{B(\rho) \backslash B\left(\rho_{0}\right)} \frac{1}{l^{2}(r)} \\
& =\left(\int_{\rho_{0}}^{\rho} \frac{d \tau}{l(\tau)}\right)^{-2}\left(\int_{\rho_{0}}^{\rho} \frac{d \tau}{l(\tau)}\right)=\left(\int_{\rho_{0}}^{\rho} \frac{d \tau}{l(\tau)}\right)^{-1} .
\end{aligned}
$$

Поскольку кривизна Риччи минимальной поверхности неположительна, то функция $l(\tau) / \tau^{n-1}$ не убывает по $\tau[19$, с. 319] и, следовательно,

$$
\begin{aligned}
& \operatorname{cap}\left(B\left(\rho_{0}\right), \partial B(\rho)\right) \leqslant\left(\int_{\rho_{0}}^{\rho} \frac{d \tau}{\tau^{n-1}}\right)^{-1} \frac{l(\rho)}{\rho^{n-1}}=\frac{(n-2) l(\rho)}{\rho^{n-1}} \cdot \frac{\left(\rho / \rho_{0}\right)^{n-2}}{\left(\rho / \rho_{0}\right)-1} \text { при } n>2, \\
& \operatorname{cap}\left(B\left(\rho_{0}\right), \partial B(\rho)\right) \leqslant \frac{l(\rho)}{\rho \log \left(\rho / \rho_{0}\right)} \text { при } n=2 .
\end{aligned}
$$

Поскольку в $\mathbb{R}^{n+1}$ всегда $\bar{S} \equiv r \equiv 0$, то из следствия 1 следует неустойчивость.

Необходимо отметить, что при $n=2$ вышеприведенный признак неустойчивости был установлен А. В. Погореловым в [20].

\section{7. Доказательство теоремы 4}

Нам потребуется

Лемма 4. Пусть $\mathscr{M}=(M, u)-$ трубка или лента с проекцией $(a, b)$ нулевой средней кривизны в искривленном лоренцевом произведении $H \times_{\delta} \widehat{\mathbb{R}}$. Тогда для всех $a<\tau_{1}<\tau_{2}<b$ виполнено

$$
\operatorname{cap}\left(\overline{M\left(a, \tau_{1}\right)}, \overline{M\left(\tau_{2}, b\right)}\right) \leqslant \frac{I}{\left(\tau_{2}-\tau_{1}\right)^{2}} \int_{\tau_{1}}^{\tau_{2}} \frac{d \tau}{\delta_{1}(\tau)},
$$

əдe

$$
I=\int_{u(M) \cap H_{\tau}} \delta(m)|\nabla f(m)|
$$

- величина, не зависящая от $\tau$, a $f(m)$ - вторая координата точки и $(m)$ в произведении $H \times_{\delta} \widehat{\mathbb{R}}$.

ДокАЗАТЕЛЬСтво. Рассмотрим функцию

$$
\varphi(m)=\frac{f(m)-\tau_{1}}{\tau_{2}-\tau_{1}}
$$

продолженную нулем на $\overline{M\left(a, \tau_{1}\right)}$ и единицей на $\overline{M\left(\tau_{2}, b\right)}$. Поскольку $\varphi(m)$ допустима в вариационной задаче (16), то

$$
\operatorname{cap}\left(\overline{M\left(a, \tau_{1}\right)}, \overline{M\left(\tau_{2}, b\right)}\right) \leqslant \int_{M\left(\tau_{1}, \tau_{2}\right)}|\nabla \varphi|^{2}=\frac{1}{\left(\tau_{2}-\tau_{1}\right)^{2}} \int_{M\left(\tau_{1}, \tau_{2}\right)}|\nabla f(m)|^{2} .
$$

Используя формулу Кронрода-Федерера [21], получим

$$
\int_{M\left(\tau_{1}, \tau_{2}\right)}|\nabla f|^{2}=\int_{\tau_{1}}^{\tau_{2}} d \tau \int_{\mathscr{M} \cap H_{\tau}}|\nabla f(m)| \leqslant \int_{\tau_{1}}^{\tau_{2}} \frac{d \tau}{\delta_{1}(\tau)} \int_{\mathscr{M} \cap H_{\tau}} \delta(m)|\nabla f(m)| .
$$


В работе [9] было показано, что функция $f(m)$ в метрике поверхности нулевой средней кривизны удовлетворяет дифференциальному уравнению

$$
\operatorname{div}(\delta(m) \nabla f(m))=0 .
$$

Применяя формулу Стокса к этому уравнению, нетрудно проверить, что величина

$$
I=\int_{\mathscr{M} \cap H_{\tau}} \delta(m)|\nabla f(m)|
$$

не зависит от $\tau$. Теперь утверждение леммы 4 следует из неравенств (25) и (26).

Лемма 5. Пусть $\mathscr{M}=(M, u)-$ поверхность нулевой средней кривизнь, удовлетворяющая условиям теоремы 4 . Тогда $k(\eta) \leqslant 0$.

ДокАЗАТЕльство. Выберем базис $\left\{E_{i}\right\}_{i=1}^{n-1}$ в касательном пространстве к поверхности $\mathscr{M}$ таким образом, чтобы $E_{1}, \ldots, E_{n-2}$ были ортогональными вектору $e$, а $E_{n-1}$ ортогонален $E_{i}, i=\overline{1, n-2}$. В таком случае

$$
\begin{aligned}
k(\eta) & =\sum_{i=1}^{n-2}\left\langle\bar{R}\left(E_{i}, \eta\right) E_{i}, \eta\right\rangle+\left\langle\bar{R}\left(E_{n-1}, \eta\right) E_{n-1}, \eta\right\rangle \\
& =-\sum_{i=1}^{n-2} K\left(E_{i}, \eta\right)+\left\langle\bar{R}\left(E_{n-1}, \eta\right) E_{n-1}, \eta\right\rangle,
\end{aligned}
$$

где $K\left(E_{i}, \eta\right)$ - секционная кривизна $H$ по двумерному направлению, определяемому векторами $E_{i}$ и $\eta$. Это равенство вытекает из формул Гаусса [19] и того, что естественное включение $H \rightarrow H \times{ }_{\delta} \widehat{\mathbb{R}}$ является вполне геодезическим вложением. Разложим вектор $E_{n-1}$ в сумму $E_{n-1}=\alpha e+X$, где $X \in T H, \alpha \in \mathbb{R}$. Имеем

$$
\begin{aligned}
\langle\bar{R}((\alpha e+X), \eta)(\alpha e+X), \eta\rangle=\alpha^{2}\langle\bar{R}(e, \eta) e, \eta\rangle & \\
& +2 \alpha\langle\bar{R}(X, \eta) e, \eta\rangle+\langle\bar{R}(X, \eta) X, \eta\rangle .
\end{aligned}
$$

В [9] доказаны равенства

$$
\bar{\nabla}_{e} e=\frac{1}{2} \bar{\nabla} \delta, \quad \bar{\nabla}_{Y} e=\frac{1}{2 \delta}\langle\bar{\nabla} \delta, Y\rangle e
$$

для всякого $Y \in T H$. Следовательно,

$$
\begin{aligned}
\langle\bar{R}(X, \eta) e, \eta\rangle= & \left\langle\bar{\nabla}_{X} \bar{\nabla}_{\eta} e-\bar{\nabla}_{\eta} \bar{\nabla}_{X}-\bar{\nabla}_{[X, \eta]} e, \eta\right\rangle \\
= & \left\langle\bar{\nabla}_{X}\left(\frac{1}{2 \delta}\langle\bar{\nabla} \delta, \eta\rangle e\right), \eta\right\rangle-\left\langle\bar{\nabla}_{\eta}\left(\frac{1}{2 \delta}\langle\bar{\nabla} \delta, X\rangle e\right), \eta\right\rangle \\
& -\frac{1}{2 \delta}\langle\bar{\nabla} \delta,[X, \eta]\rangle\langle e, \eta\rangle=0
\end{aligned}
$$

в силу того, что $\langle e, \eta\rangle=0$.

Далее,

$$
\begin{aligned}
\langle\bar{R}(e, \eta) e, \eta\rangle & =\left\langle\bar{\nabla}_{e} \bar{\nabla}_{\eta} e, \eta\right\rangle-\left\langle\bar{\nabla}_{\eta} \bar{\nabla}_{e} e, \eta\right\rangle-\left\langle\bar{\nabla}_{[e, \eta]} e, \eta\right\rangle \\
& =\left\langle\bar{\nabla}_{e}\left(\frac{1}{2 \delta}\langle\bar{\nabla} \delta, \eta\rangle e\right), \eta\right\rangle-\frac{1}{2}\left\langle\bar{\nabla}_{\eta} \bar{\nabla} \delta, \eta\right\rangle \\
& =\frac{1}{4 \delta}\langle\bar{\nabla} \delta, \eta\rangle\langle\bar{\nabla} \delta, \eta\rangle-\frac{1}{2}\left\langle\bar{\nabla}_{\eta} \bar{\nabla} \delta, \eta\right\rangle \\
& =-\frac{1}{2} \sqrt{\delta}\left\langle\nabla_{\eta} \nabla \sqrt{\delta}, \eta\right\rangle \leqslant 0
\end{aligned}
$$


в силу выпуклости функции $\sqrt{\delta}$. Таким образом, из $(27)-(29)$ и неотрицательности секционной кривизны многообразия $H$ получается требуемое.

Для доказательства теоремы 4 мы применим леммы $3-5$ и теорему 3 , что даст $\left\|A^{\eta}\right\|^{2}-\|\nabla \eta\|^{2} \equiv 0$. Следовательно, $\left\|A^{\eta}\right\| \equiv 0$ и поверхность $\mathscr{M}$ горизонтально вполне геодезическая.

ДОКАЗАТЕЛЬСТВО СЛЕДСТВИЯ 3 проводится аналогичными рассуждениями для искривленного риманова произведения с использованием признака параболичности (23). Именно, условие (23) обеспечит неположительность величины $k(\eta)$ и мы, используя леммы 3 и 4 , получим

$$
\begin{aligned}
Q^{\eta}\left(h_{0}\right) & =\int_{\mathscr{M}}\left\{\left|\nabla h_{0}\right|^{2}-h_{0}^{2}\left(-\|\nabla \eta\|^{2}+\left\|A^{\eta}\right\|^{2}-k(\eta)\right)\right\} \\
& \leqslant \int_{\mathscr{M}}\left\{\left|\nabla h_{0}\right|^{2}-h_{0}^{2}\left\|A^{\eta}\right\|^{2}\right\} \leqslant 0 .
\end{aligned}
$$

ДоКАЗАТЕЛЬСТво СЛЕДСТВИЯ 2. На основании следствия 3 заключаем, что $\bar{\nabla} \eta \equiv 0$. Поэтому $\eta \equiv \eta_{0}=$ const и, значит, $u(M)=\mathscr{M}$ лежит во времениподобной гиперплоскости, ортогональной вектору $\eta_{0}$.

\section{8. Доказательство теоремы 5}

В качестве первого шага, докажем следуюшее вспомогательное утверждение.

ЛЕмма 6. Пусть в полосе

$$
\Pi=\{(x, y): 0<y<1\}
$$

задана гармоническая функиия $и(x, y)$ с краевым условием

$$
\left.\frac{\partial u}{\partial y}\right|_{\partial \Pi}=0 .
$$

Ecлu

$$
|u(x, y)| \leqslant c(|x|+1)
$$

для некоторой постоянной $c \geqslant 0$, то и - линейная функция.

ДокАЗАТЕЛЬСТво. Пусть

$$
h(x)=\int_{0}^{1} u^{2}(x, y) d y .
$$

Тогда

$$
\begin{aligned}
h^{\prime}(x) & =2 \int_{0}^{1} u u_{x} d y \\
h^{\prime \prime}(x) & =2 \int_{0}^{1} u_{x}^{2}+2 \int_{0}^{1} u u_{x x} d y=2 \int_{0}^{1} u_{x}^{2}-2 \int_{0}^{1} u u_{y y} d y .
\end{aligned}
$$

В силу краевого условия после интегрирования по частям получаем

$$
h^{\prime \prime}(x)=2 \int_{0}^{1}\left(u_{x}^{2}+u_{y}^{2}\right) d y .
$$


$\mathrm{K}$ производной функции $h(x)$ применим неравенство Коши

$$
h^{\prime 2}(x) \leqslant 4 \int_{0}^{1} u^{2} d y \int_{0}^{1} u_{x}^{2} d y=4 h(x)\left\{\frac{1}{2} h^{\prime \prime}(x)-\int_{0}^{1} u_{y}^{2} d y\right\}
$$

В полученном дифференциальном неравенстве сделаем замену $u^{2}(x)=h(x)$. Тогда оно примет вид

$$
u^{\prime \prime} u \geqslant a^{2}(x)
$$

где $a^{2}(x)=\int_{0}^{1} u_{y}^{2} d y$. Заметим, что функция $u(x)$ выпукла вниз, и поэтому в силу условия на рост $u(x)$ получаем

$$
u^{\prime \prime}(x) \geqslant \frac{a^{2}(x)}{c(1+|x|)}
$$

Далее, функция $u_{y}(x, y)$ также является гармонической в полосе П, причем с нулевыми граничными значениями. Повторяя вышеприведенные рассуждения, делаем вывод, что функция $a(x)$ является выпуклой. Значит, не ограничивая общности, можно считать, что если $a(x)$ не является тождественным нулем, то сушествует $x_{0}>0$, для которого $a(x) \geqslant a\left(x_{0}\right)>0$. Поэтому при $x \geqslant x_{0}$ выполнено

$$
u^{\prime \prime}(x) \geqslant \frac{a^{2}\left(x_{0}\right)}{c(1+x)}
$$

Интегрируя это неравенство два раза, получим

$$
\max _{0<y<1}|u(x, y)| \geqslant u(x) \geqslant u\left(x_{0}\right)+u^{\prime}\left(x_{0}\right)\left(x-x_{0}\right)+\frac{a^{2}\left(x_{0}\right)}{c}(1+x)(\log (1+x)-1),
$$

что, очевидно, противоречит ограничению на рост функции $u(x, y)$. Поэтому $a(x) \equiv 0$ и функция $u(x, y)$ зависит только от $x$ и является линейной. Лемма доказана.

ДОКАЗАТЕЛЬСТво ТЕОРЕМЫ. В силу односвязности ленты для функции $f(m)$ сушествует сопряженная гармоническая функция $g(m)$, причем голоморфное отображение $G: \mathscr{M} \rightarrow \Pi=\frac{1}{I}(f(m), g(m))$ является взаимно однозначньм (см. [22]). Здесь

$$
I=\int_{E_{t}}|\nabla f| .
$$

Тогда для гармонической функции $u(x, y)=\varphi\left(G^{-1}(x, y)\right)$ выполнены условия леммы 4. Из этого делаем вывод, что $\varphi$ линейно зависит от $f(m)$, а поверхность $\mathscr{M}$ есть часть геликоида. Теорема доказана. 


\section{Список литературы}

1. Атырбеков A., Соколов Д. Д. Геометрия в целом в плоском пространстве-времени. Ташкент: Изд-во “ФАН" Узбекской ССР, 1991.

2. Горох В. П. Об устойчивости минимальной поверхности в псевдоевклидовом пространстве // Укр. геом. сб. 1990. №33. С. 41-46.

3. Shen $Y$. On maximal submanifolds in pseudo-Riemannian manifolds // J. Hang-zhou Univ. Natur. Sci. Ed. 1991. V. 18. № 7. P. 371-376.

4. Барбашов Б. М., Нестеренко В. В. Модель релятивистской струны в физике адронов. М.: Энергоатомиздат, 1987.

5. Клячин B.A., Миклюков B. М. Об одном емкостном признаке неустойчивости минималњных гиперповерхностей // ДАН. 1993. Т. 330. № 4. С. 424-426.

6. Бим Джс., Эрлих П. Глобальная лоренцева геометрия. М.: Мир, 1985.

7. Кобаяси ШІ., Номидзу К. Основы дифференциальной геометрии. Т. 2. М.: Наука, 1981.

8. Соболев С. Л. Некоторые применения функционального анализа в математической физике. Новосибирск: Изд-во СО АН СССР, 1962.

9. Клячин B.A., Миклюков В.M. Условия конечности времени существования максимальных трубок и лент в искривленных лоренцевых произведениях // Изв. РАН. Сер. матем. 1994. Т. 58. №3. С. 196-210.

10. Клячин B. A. Максимальные трубчатые поверхности произвольной коразмерности в пространстве Минковского // Изв. РАН. Сер. матем. 1993. Т. 57. № 4. С. 118-131.

11. Тужилин A. А. Индексы типа Морса двумерных минимальных поверхностей в $\mathbb{R}^{3}$ и $\mathrm{H}^{3}$ // Изв. АН СССР. Сер. матем. 1991. Т. 55. № 2. С. 581-607.

12. Лосев А. Г. Некоторые лиувиллевы теоремы на римановых многообразиях специального вида // Изв. вузов. Математика. 1991. № 12. С. 15-24.

13. Гольдитейн В. М., Решетняк Ю. Г. Введение в теорию функций с обобщенными производными и квазиконформные отображения. М.: Наука, 1983.

14. Vuorinen M. Conformal Geometry and Quasiregular Mappings // Lect. Notes in Math. V. 1319. Berlin: Springer-Verlag, 1988.

15. do Carmo M., Peng C. K. The stable minimal surfaces in $\mathbb{R}^{3}$ are planes // Bull. (Ne Ser.) Amer. Math. Soc. 1979. V. 1. P. 903-906.

16. Миклюков В. М. Минимальные ленты типа геликоида // IX-я Всесоюзн. геом. конференция. Кишинев, 1988. С. 213.

17. Simons J. Minimal varieties in riemannian manifolds // Ann. of Math. 1986. V. 88. № 2. P. 62-105.

18. Бессе А. Многообразия Эйнштейна. Т. 1. М.: Мир, 1990.

19. Бишоп P., Криттенден P. Геометрия многообразий. М.: Мир, 1967.

20. Погорелов А.В. Об устойчивости минимальных поверхностей // ДАН СССР. 1981. T. 260. № 2. C. 293-295.

21. Бураго Ю. Д., Залгаллер В. А. Геометрические неравенства. Л.: Наука, 1980.

22. Миклюков В. М. Максималњыые трубки и ленты в пространстве Минковского // Матем. сб. 1992. Т. 183. № 12. С. 45-76.

Волгоградский государственный университет

E-mail: klchnv@math.vgu.tsaritsyn.su;

Поступила в редакцию

miklukov@math.vgu.tsaritsyn.su 